\title{
Free light chains kappa can differentiate between myelitis and noninflammatory myelopathy
}

Marie Süße, MD, Fritz Feistner, Matthias Grothe, MD, Matthias Nauck, MD, Alexander Dressel, MD, and Malte Johannes Hannich, MD

Neurol Neuroimmunol Neuroinflamm 2020;7:e892. doi:10.1212/NXI.0000000000000892

\section{Abstract}

\section{Objective}

To test the hypothesis that the intrathecal synthesis of free light chain kappa (FLC-k) can be used as a CSF biomarker to differentiate patients with myelitis due to multiple sclerosis (MS), myelitis due to neuromyelitis optica spectrum disease (NMOSD), and noninflammatory myelopathy, we analyzed FLC-k in 26 patients with MS myelitis, 9 patients with NMOSD myelitis, and 14 patients with myelopathy.

\section{Methods}

This is a retrospective monocentric cohort study. FLC-k were analyzed using the nephelometric Siemens FLC-k kit in paired samples of CSF and sera. Intrathecal fraction (IF) of FLC-k was plotted in a FLC-k quotient diagram.

\section{Results}

Ninety-six percent of patients with MS myelitis had an intrathecal synthesis of FLC-k in comparison with $55.6 \%$ for NMOSD and $14.3 \%$ of patients with noninflammatory myelopathy. The locally synthesized absolute amount of FLC-k was significantly higher in patients with myelitis due to MS than in patients with NMOSD $(p=0.038)$ or noninflammatory myelopathy $(p<0.0001)$. The sensitivity of FLC-k synthesis to detect inflammation in patients with myelitis is $85.7 \%$. Using a receiver operating characteristic analysis, FLC-k IF $>78 \%$ can discriminate patients with myelitis due to MS and NMOSD with a sensitivity of $88.5 \%$ and a specificity of $88.9 \%$

\section{Conclusions}

With the hyperbolic reference range in quotient diagrams for FLC-k, it is possible to distinguish inflammatory myelitis from noninflammatory myelopathies. An FLC-k IF $>78 \%$ can be a hint to suspect myelitis due to MS rather than NMOSD.

\author{
Correspondence \\ Dr. Süße \\ marie.suesse@uni-greifswald.de
}




\section{Glossary}

CIS = clinically isolated syndrome; FLC-k = free light chain kappa; IF = intrathecal fraction; Ig = immunoglobulin; NMOSD = neuromyelitis optica spectrum disease; $\mathbf{O C B}=$ oligoclonal band; $\mathbf{R O C}=$ receiver operating characteristic.

The causes of myelopathies can be manifold, and there is a broad range of differential diagnoses. Besides compressive myelopathy, the most frequent causes of myelopathy are inflammatory disorders such as MS or neuromyelitis optica spectrum disease (NMOSD). ${ }^{1}$ Although neuroimaging is essential for the evaluation of myelopathy, overlap in the imaging appearance is a challenging issue. ${ }^{1}$ The additional analysis of CSF can provide useful information on further etiologic determination. $^{1-3}$ In noninflammatory myelopathies, CSF analysis seldom reveals signs of inflammation, such as pleocytosis or intrathecal immunoglobulin (Ig) G synthesis. ${ }^{3}$ In MS, there is a high prevalence of oligoclonal band (OCB) in approximately $95 \%,{ }^{4}$ in contrast to NMOSD, where the absence of OCB is a supportive evidence for the correct diagnosis, although sensitivity and specificity are modest. ${ }^{5}$ It has been shown that the analysis of free light chain kappa (FLC-k) can be used in the diagnostic process of MS with equal sensitivity to OCB analysis. ${ }^{6}$ Methodologically, its use provides the advantage of an easyto-use, commercially available nephelometric assay for a rapid and quantitative evaluation of intrathecal inflammation. ${ }^{7} \mathrm{Up}$ to date, no study analyzed the diagnostic performance of FLC-k intrathecal synthesis for the discrimination between patients with MS myelitis, NMOSD myelitis, and noninflammatory myelopathies. The prespecified hypothesis stated that the in trathecal synthesis of FLC-k in CSF is the highest in patients with MS associated myelitis, lower in myelitis due to NMOSD, and absent in non-inflammatory myelopathies.

\section{Methods}

This is a retrospective monocentric cohort study. Patients were identified for analysis based on diagnosis in medical records. Paired CSF and serum samples were acquired between 2008 and 2020 from patients of the Department of Neurology, University Medicine Greifswald, Germany. Between 2008 and 2016 samples were stored at $-80^{\circ} \mathrm{C}$. The samples acquired between 2016 and 2020 have been measured either as part of other study cohorts $^{7-9}$ or for the purpose of this study without being stored. Patients were grouped by the clinical diagnosis according to the corresponding criteria (MS: modified Mc Donald criteria ${ }^{10}$; NMOSD: criteria proposed by Wingerchuk et al. ${ }^{5}$ ).

\section{Laboratory analysis}

Laboratory analyses were performed in the Interdisciplinary CSF laboratory of the University Medicine Greifswald as described previously.,11

Table 1 Baseline characteristics and cerebrospinal fluid results

\begin{tabular}{|c|c|c|c|c|}
\hline & Myelitis due to MS or CIS & NMOSD & Noninflammatory myelopathy ${ }^{a}$ & $p$ Value $^{\mathrm{b}}$ \\
\hline n (\%) & $26(53.1)$ & $9(18.4)$ & $14(28.6)$ & \\
\hline Age, y & $35.5(29.75 ; 49.25)$ & $56(47.5 ; 63)$ & $58(46.25 ; 74)$ & $<0.0001$ \\
\hline Female, n (\%) & $15(43.3)$ & $5(44.4)$ & $7(50)$ & 0.926 \\
\hline Immunotherapy, n (\%) & 0 & $4(44.4)^{c}$ & & \\
\hline QAlb & $5(4.13 ; 7.19)$ & $17.02(4.495 ; 24.12)$ & $10.58(7.09 ; 12.1)$ & 0.001 \\
\hline $\mathrm{CC}, / \mu \mathrm{L}$ & $9(3 ; 21)$ & $2(1 ; 4)$ & $1(1 ; 2)$ & 0.001 \\
\hline FLC-k serum, mg/L & $11.8(9.85 ; 14.8)$ & $17.9(15.3 ; 36.15)$ & $13.8(9.67 ; 23.35)$ & 0.006 \\
\hline FLC-k CSF, mg/L & $4.22(1.29 ; 7.92)$ & $1.04(0.43 ; 8.88)$ & $0.39(0.15 ; 0.72)$ & 0.0001 \\
\hline FLC-k index & $63.71(19.39 ; 154.999)$ & $5.81(1.07 ; 13.35)$ & $2.05(1.43 ; 2.52)$ & $<0.0001$ \\
\hline FLC-k IF, \% & $94.8(84.2 ; 97.9)$ & $34.5(0 ; 76.6)$ & $0(0 ; 0)$ & $<0.0001$ \\
\hline OCB CSF, n (\%) & $23(88.5)$ & $3(33.3)$ & $0(0)$ & $<0.0001$ \\
\hline
\end{tabular}

Abbreviations: $\mathrm{Ab}=$ antibody; $\mathrm{CC}=$ cell count; $\mathrm{CIS}=$ clinically isolated syndrome; FLC-k = free light chains kappa; IF = intrathecal fraction; neg = negative; NMOSD = neuromyelitis optica spectrum disease; OCB = oligoclonal band; pos = positive; QAlb = albumin quotient; SCT = stem cell transplantation. Continuous data are expressed as median (1st; 3rd quartile); nominal data are given as percentages.

${ }^{a}$ Compressive myelopathy $(n=6)$, vascular myelopathy $(n=5)$, radiation myelopathy $(n=1)$, and unknown etiology, but no signs of inflammation either in CSF nor in MRI $(n=2)$.

b Statistical significance $p$ value $\leq 0.05$.

' Immunotherapy patient 1: anti-AOP-4 Ab pos/OCB pos/IF FLC-k $75.6 \%$ rituximab 9 months before CSF analysis; patient 2: anti AQP-4 Ab pos/OCB neg/IF FLC-k 34.5\% Allo-SCT 16 months before CSF analysis; patient 3: anti-AQP-4 Ab pos/OCB neg/IF FLC-k 0 rituximab 2 months before CSF analysis; patient 4: antiAQP-4 Ab pos/OCB neg/IF FLC-k 49.1\% methylprednisolone $1 \mathrm{mg} / \mathrm{kg}$ per os at the time of CSF analysis. 

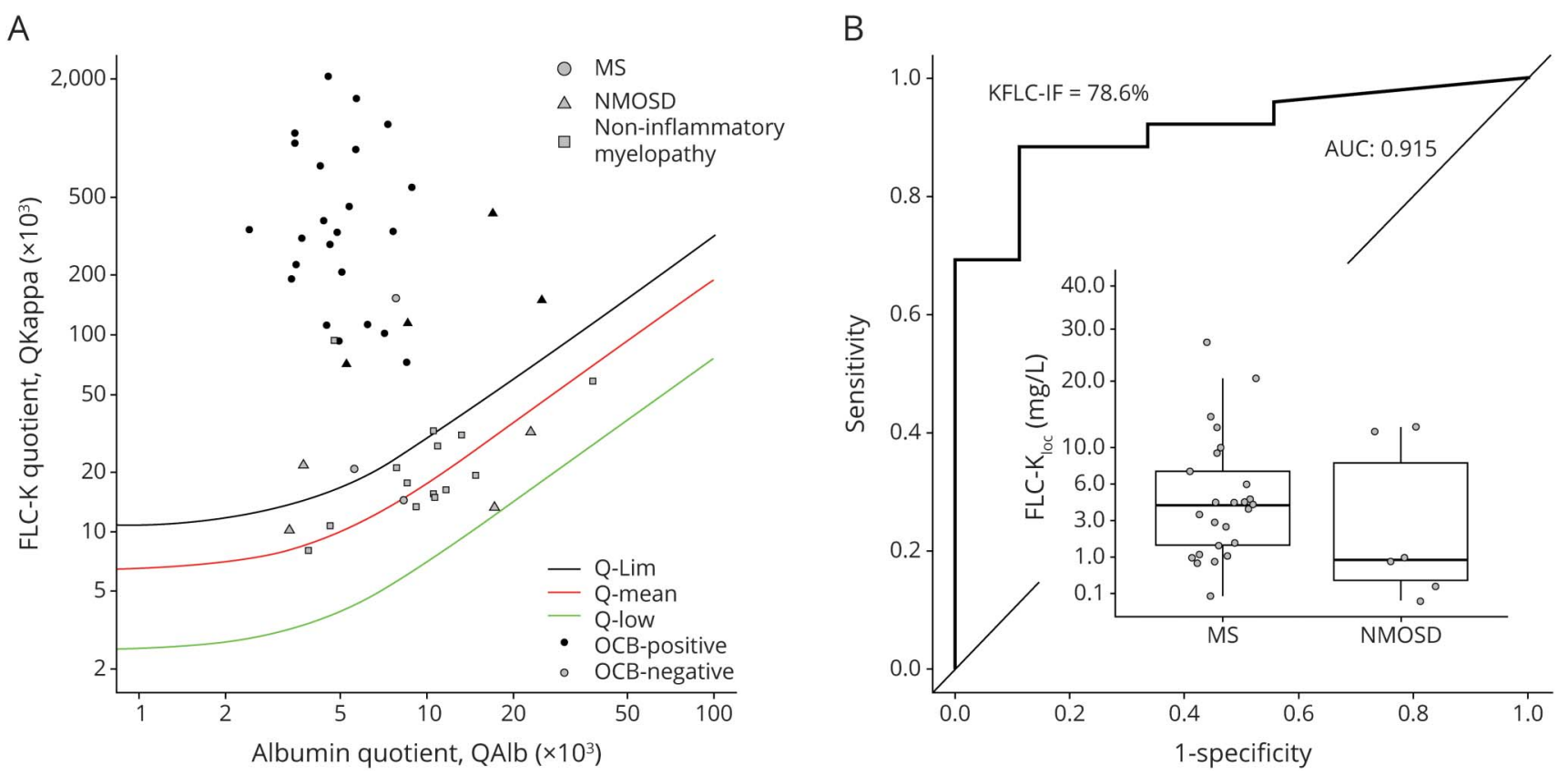

(A) Data of the FLC-k quotients in a double logarithmic FLC-k Reibergram. The black line shows $\mathrm{Q}_{\mathrm{FLC}-\mathrm{k}}(\mathrm{lim})$; the red line shows the $\mathrm{Q}_{\mathrm{FLC}-\mathrm{k}}(\mathrm{mean})$. The green line is the lower limit of the reference range QfLC-k(low). Ninety-six percent of the FLC-k quotients in patients with myelitis due to MS or CIS are above the upper discrimination line $\mathrm{Q}_{\mathrm{lim}}$. Fifty-five of the patients with a myelitis due to NMOSD showed FLC-k quotients $>\mathrm{Q}_{\text {lim. }}$. Approximately $85.7 \%$ of patients with a noninflammatory myelopathy had FLC-k values $<\mathrm{Q}_{\lim }$. (B) ROC analysis in respect to FLC-IF $>\mathrm{Q}_{\text {lim. }}$. The AUC is 0.915 . With a cut-off of $78.6 \% \mathrm{IF}$, sensitivity is $88.5 \%$, specificity $88.9 \%$ to discriminate patients with MS/CIS and NMOSD. Box plots: The locally synthesized absolute amount of $F L C-k\left(K_{\text {loc }}=\left[Q_{k a p p a}\right.\right.$ (total) $\left.\left.\mathrm{Q}_{\mathrm{kapp}}(\mathrm{mean})\right] \times \mathrm{S}_{\mathrm{kappa}}[\mathrm{mg} / \mathrm{L}]\right)$ is significantly higher in patients with myelitis due to MS than in patients with NMOSD $(p=0.038)$. AUC $=$ area under the curve; $\mathrm{CIS}=$ clinically isolated syndrome; FLC-k = free light chains kappa; IF = intrathecal fraction; NMOSD = neuromyelitis optica spectrum disease; OCB = oligoclonal band; $\mathrm{Q}=$ quotient; $\mathrm{ROC}=$ receiver operating characteristic.

FLC-k in sera and CSF were measured by nephelometry with the N Latex FLC kappa kit (Siemens Healthcare Diagnostics Products GmbH, Marburg, Germany) according to the manufacturers protocol on the BN Prospec analyzer. CSF predilution was set to $1: 1$; serum predilution was set to $1: 100$. The lower limit of quantification was $0.034 \mathrm{mg} / \mathrm{L}$ and was given by the manufacturer (details are provided in table e- 1 , links.lww.com/NXI/A316).

The hyperbolic reference range and the amount of intrathecal synthesized FLC-k was calculated according to the formulas defined by Reiber et al. ${ }^{11}$

\section{Statistical analysis}

SPSS 25.0 (IBM Co., Armonk, NY) and RStudio (R version 3.5.1 2018-07-02) were used for statistical and graphical processing of the data. Statistical significance was assessed using $\chi^{2}$ test or Fisher exact test for nominal data. Intergroup comparison was performed using the Kruskal-Wallis analysis of ranks test. For statistical group comparison, the FLC-k intrathecal fraction (IF) and the locally synthesized absolute amount of FLC-k was calculated in relation to the $\mathrm{Q}_{\text {mean }}$. The sensitivity and specificity of FLC-k IF was displayed in a receiver operating characteristic (ROC) curve. The optimal cut-off to discriminate patients with myelitis due to MS and
NMOSD was determined using the Youden index. $p$ Values $\leq 0.05$ were regarded as statistically significant.

\section{Standard protocol approvals, registrations, and patient consents}

The study has been approved by the institutional review board (BB019/18).

\section{Data availability}

Anonymized data will be shared by request from any qualified investigator.

\section{Results}

Forty-nine patients were retrospectively identified for analysis. Twenty-six patients $(53.1 \%)$ with myelitis as manifestation of MS/clinically isolated syndrome (CIS), 9 patients (18.4\%) with a myelitis due to NMOSD, and 14 patients with a noninflammatory myelopathy $(28.6 \%)$ (table 1$)$.

\section{FLC-k values in quotient diagrams}

Figure 1A shows the IF of FLC-k values in quotient diagrams. Ninety-six percent $(n=25 / 26)$ of the FLC-k quotients in patients with myelitis due to MS/CIS are above the upper discrimination line $\mathrm{Q}_{\lim }$ representing intrathecal FLC-k 


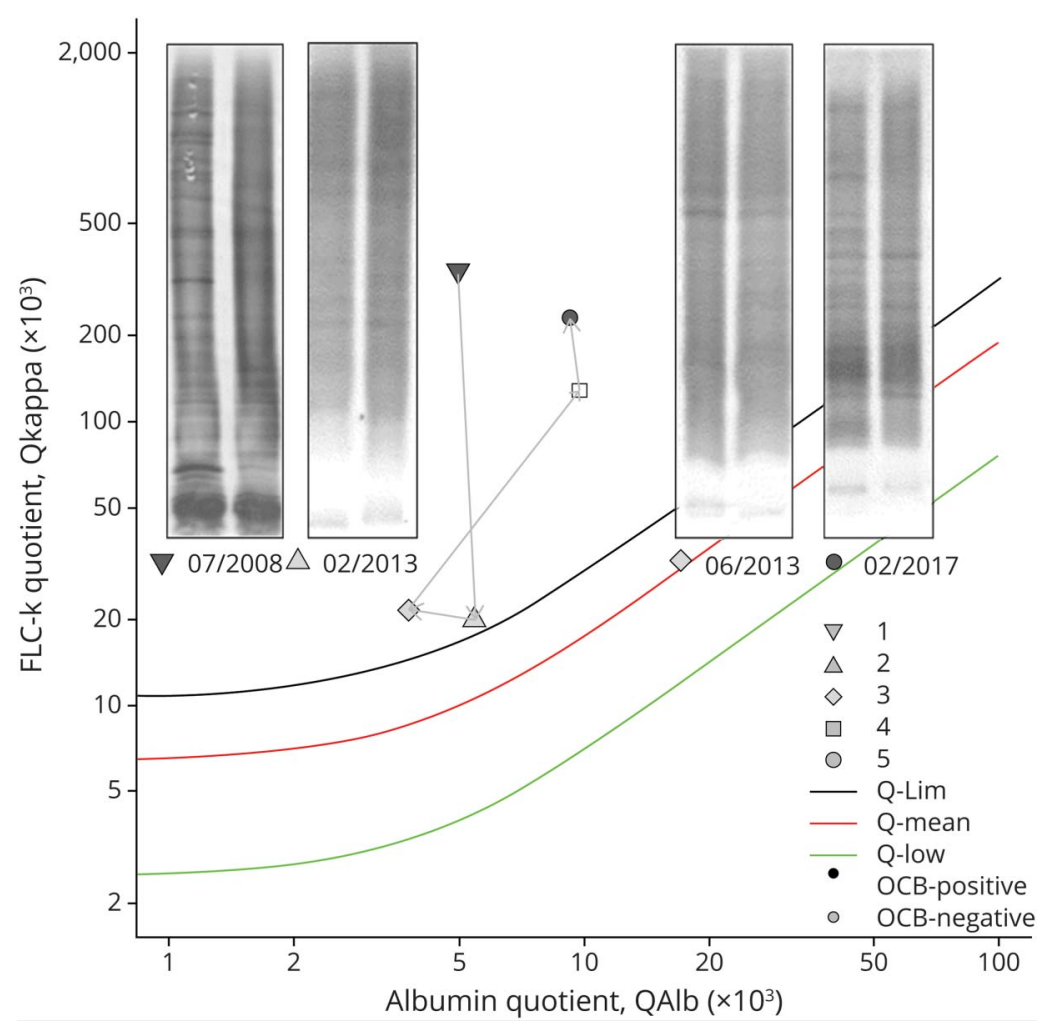

See also the legend of figure 1. Inverted triangle: CSF analysis 07/2008 before immunotherapy, IF 95.1\%; triangle: CSF analysis 02/2013 12 months after autologousSCT, IF 12.5\%; square: CSF analysis 06/2013, IF 34.5\%; rectangle CSF analysis 02/2017, IF 77.8; circle CSF analysis 02/2017 IF 88.3\% (new disease activity). FLC-k = free light chains kappa; IF = intrathecal fraction; neg = negative; NMOSD = neuromyelitis optica spectrum disease; OCB = oligoclonal band; pos = positive; $\mathrm{Q}$ = quotient; $\mathrm{SCT}=$ stem cell transplantation. synthesis. Sensitivity for OCB detection in CSF in this cohort is $88.5 \%$.

In contrast to myelitis due to MS/CIS, only 5 of 9 (55.6\%) patients diagnosed with a NMOSD myelitis showed intrathecal FLC-k synthesis (table 1, figure 1). Of interest is the clinical course of 1 patient whose OCB became transiently negative after stem cell transplantation, although FLC-k quotients remained $>\mathrm{Q}_{\lim }$ (figure 2).

The sensitivity of FLC-k synthesis to detect inflammation in patients with myelitis (due to MS/CIS and NMOSD) is $85.7 \%$, in comparison with $74.3 \%$ for OCB detection.

All patients diagnosed with a noninflammatory myelopathy were OCB negative and 2 had FLC-k values $>Q_{\lim }(14.3 \%)$.

\section{The total amount of intrathecal FLC-k synthesis discriminates MS myelitis from NMOSD myelitis}

The locally synthesized absolute amount of FLC-k is significantly higher in patients with MS myelitis than in patients with NMOSD myelitis $(p=0.038)$ or noninflammatory myelopathy $(p<0.0001)$ in post hoc analysis. Using a ROC analysis, FLC-k IF $>78.6 \%$ can discriminate between patients with myelitis due to MS and NMOSD with a sensitivity of $88.5 \%$ and a specificity of $88.9 \%$ (figure 1B).

\section{Discussion}

The determination of FLC-k and interpretation in quotient diagrams ${ }^{11}$ reached a sensitivity of $96 \%$ to confirm intrathecal inflammation in patients with a MS myelitis in comparison with $55.6 \%$ in patients with NMOSD myelitis and $14.3 \%$ in patients with noninflammatory myelopathies. Sensitivity to detect intrathecal inflammation in both cohorts of inflammatory myelitis (MS/CIS and NMOSD) was higher than the OCB analysis as the current gold standard (88.5\% for myelitis due to MS/CIS and $33.3 \%$ for NMOSD, respectively).

One explanation is that FLC-k probably represents other aspects of inflammation, for example higher values of FLC-k can also reflect intrathecal IgM or A synthesis in contrast to OCB which represents IgG.

Another aspect is the analytical sensitivity of OCB detection in cases with low intrathecal IgG synthesis. As evidenced by case studies with no or small amounts of OCB bands in isoelectric focusing, ${ }^{8}$ patients with inflammatory diseases and negative OCB still present an FLC-k IF $>0 \%$.

The only other study so far describing FLC-k values to discriminate patients with MS and NMOSD obtained comparable results with our study cohort. ${ }^{12}$ Although using the index method for FLC-k interpretation with the known risk of false 
positive or negative values ${ }^{11}$ and not restricting the cohort of MS to myelitis manifestation, the highest FLC-k index values were seen in patients with MS in comparison to NMOSD. ${ }^{12}$ An advantage of FLC- $k$ analysis is the quantification in comparison with the qualitative OCB analysis. We could identify an IF of $78.6 \%$ with a sensitivity of $88.9 \%$ and a specificity of $88.5 \%$ to discriminate patients with myelitis due to MS and NMOSD.

One major limitation of this study is the small sample size of the patient and the control cohort due to the rarity of the described diagnoses. The proposed FLC-k IF to discriminate patients with MS myelitis and NMOSD myelitis has to be validated preferably in a prospective multicenter study with a larger cohort.

With the hyperbolic reference range in quotient diagrams for FLC-k, it is possible to distinguish inflammatory myelitis from noninflammatory myelopathies. The additional measurement of FLC-k can support the diagnosis in patients with a suspected inflammatory origin of myelitis. A FLC-k IF $>78 \%$ can be a hint to suspected MS myelitis rather than NMOSD myelitis in clinically unclear cases, in consideration of other surrounding diagnostic results, such as MRI and antibody status.

\section{Study funding}

This research received no specific grant from any funding agency in the public, commercial, or not-for-profit sectors.

\section{Disclosure}

M. Süße and F. Feistner have nothing to disclose. M. Grothe obtained travel reimbursement and speaker's honoraria from Novartis Pharma, Teva Pharmaceuticals, Merck, Sanofi Genzyme, and Biogen Idec as well as research grants from the Federal Ministry for Research and Education in Germany. M. Nauck reports nonfinancial support by Siemens Healthineers, The Binding Site Group, Becton Dickinson, DZHK (German Centre for Cardiovascular Research, Partner Site Greifswald, University Medicine, Greifswald, Germany), DGKL (German Federation of Clinical Chemistry and Laboratory Medicine), German Federal Medical Association, Roche Diagnostics Germany GmbH; he also received personal fees by Boehringer Ingelheim and Becton Dickinson; Grant by DZHK, LVL technologies, Bruker BioSpin, Abbott, Radiometer, Tosoh and IDS Immunodiagnostic Systems Deutschland GmbH. A. Dressel reports speaker honoraria and travel reimbursement to his institution from Siemens Healthineers. M.J. Hannich has nothing to disclose. Go to Neurology.org/NN for full disclosures.

\section{Publication history}

Received by Neurology: Neuroimmunology \& Neuroinflammation April 29, 2020. Accepted in final form August 17, 2020.
Appendix Authors

\begin{tabular}{lll}
\hline Name & Location & Contribution \\
\hline $\begin{array}{l}\text { Marie } \\
\text { Süße, MD }\end{array}$ & $\begin{array}{l}\text { Department of Neurology, } \\
\text { University Medicine }\end{array}$ & $\begin{array}{l}\text { Designed and } \\
\text { conceptualized the } \\
\text { Greifswald, Germany }\end{array}$ \\
& $\begin{array}{l}\text { study, analyzed the data, } \\
\text { drafted the manuscript, } \\
\text { and revised the } \\
\end{array}$ & manuscript for \\
& intellectual content
\end{tabular}

\begin{tabular}{|c|c|c|}
\hline $\begin{array}{l}\text { Fritz } \\
\text { Feistner }\end{array}$ & $\begin{array}{l}\text { Department of Neurology, } \\
\text { University Medicine } \\
\text { Greifswald, Germany }\end{array}$ & $\begin{array}{l}\text { Major role in the } \\
\text { acquisition of data and } \\
\text { analyzed the data }\end{array}$ \\
\hline $\begin{array}{l}\text { Matthias } \\
\text { Grothe, MD }\end{array}$ & $\begin{array}{l}\text { Department of Neurology, } \\
\text { University Medicine } \\
\text { Greifswald, Germany }\end{array}$ & $\begin{array}{l}\text { Revised the manuscript } \\
\text { for intellectual content }\end{array}$ \\
\hline $\begin{array}{l}\text { Matthias } \\
\text { Nauck, MD }\end{array}$ & $\begin{array}{l}\text { Institute of Clinical Chemistry } \\
\text { and Laboratory Medicine, } \\
\text { University Medicine } \\
\text { Greifswald, Germany }\end{array}$ & $\begin{array}{l}\text { Revised the manuscript } \\
\text { for intellectual content }\end{array}$ \\
\hline $\begin{array}{l}\text { Alexander } \\
\text { Dressel, MD }\end{array}$ & $\begin{array}{l}\text { Department of Neurology, } \\
\text { Carl-Thiem-Klinikum Cottbus, } \\
\text { Germany }\end{array}$ & $\begin{array}{l}\text { Revised the manuscript } \\
\text { for intellectual content }\end{array}$ \\
\hline $\begin{array}{l}\text { Malte } \\
\text { Johannes } \\
\text { Hannich, } \\
\text { MD }\end{array}$ & $\begin{array}{l}\text { Institute of Clinical Chemistry } \\
\text { and Laboratory Medicine, } \\
\text { University Medicine } \\
\text { Greifswald, Germany }\end{array}$ & $\begin{array}{l}\text { Designed and } \\
\text { conceptualized the } \\
\text { study, analyzed the data, } \\
\text { and revised the } \\
\text { manuscript for } \\
\text { intellectual content }\end{array}$ \\
\hline
\end{tabular}

\section{References}

1. Weidauer S, Wagner M, Nichtweiß M. Magnetic resonance imaging and clinical features in acute and subacute myelopathies. Clin Neuroradiol 2017;27: 417-433.

2. Jarius S, Paul F, Franciotta D, et al. Cerebrospinal fluid findings in aquaporin-4 antibody positive neuromyelitis optica: results from 211 lumbar punctures. J Neurol Sci 2011;306:82-90

3. Barreras P, Fitzgerald KC, Mealy MA, et al. Clinical biomarkers differentiate myelitis from vascular and other causes of myelopathy. Neurology 2018;90: e12-e21.

4. Dobson R, Ramagopalan S, Davis A, Giovannoni G. Cerebrospinal fluid oligoclonal bands in multiple sclerosis and clinically isolated syndromes: a meta-analysis of prevalence, prognosis and effect of latitude. J Neurol Neurosurg Psychiatry 2013;84: 909-914.

5. Wingerchuk DM, Banwell B, Bennett JL, et al. International consensus diagnostic criteria for neuromyelitis optica spectrum disorders. Neurology 2015;85: 177-189.

6. Leurs CE, Twaalfhoven H, Lissenberg-Witte BI, et al. Kappa free light chains is a valid tool in the diagnostics of MS: a large multicenter study. Mult Scler 2020;26: 912-923.

7. Süße M, Hannich M, Petersmann A, et al. Kappa free light chains in cerebrospinal fluid to identify patients with oligoclonal bands. Eur J Neurol 2018;25: 1134-1139.

8. Süße M, Feistner F, Holbe C, et al. Diagnostic value of kappa free light chains in patients with one isolated band in isoelectric focusing. Clin Chim Acta 2020;507: 205-209.

9. Süße M, Reiber H, Grothe M, et al. Free light chain kappa and the polyspecific immune response in MS and CIS: application of the hyperbolic reference range for most reliable data interpretation. J Neuroimmunol 2020;346:577287.

10. Thompson AJ, Banwell BL, Barkhof F, et al. Diagnosis of multiple sclerosis: 2017 revisions of the McDonald criteria. Lancet Neurol 2018;17:162-173.

11. Reiber H, Zeman D, Kušnierová P, Mundwiler E, Bernasconi L. Diagnostic relevance of free light chains in cerebrospinal fluid: the hyperbolic reference range for reliable data interpretation in quotient diagrams. Clin Chim Acta 2019;497: 153-162.

12. Cavalla P, Caropreso P, Limoncelli S, et al. Kappa free light chains index in the differential diagnosis of multiple sclerosis from neuromyelitis optica spectrum disorders and other immune-mediated central nervous system disorders. J Neuroimmunol 2020;339:577122. 


\title{
Neurology ${ }^{\oplus}$ \\ Neuroimmunology \& Neuroinflammation
}

\author{
Free light chains kappa can differentiate between myelitis and noninflammatory \\ myelopathy \\ Marie Süße, Fritz Feistner, Matthias Grothe, et al. \\ Neurol Neuroimmunol Neuroinflamm 2020;7; \\ DOI 10.1212/NXI.0000000000000892
}

This information is current as of September 18, 2020

Neurol Neuroimmunol Neuroinflamm is an official journal of the American Academy of Neurology.

Published since April 2014, it is an open-access, online-only, continuous publication journal. Copyright

Copyright (C) 2020 The Author(s). Published by Wolters Kluwer Health, Inc. on behalf of the American

Academy of Neurology.. All rights reserved. Online ISSN: 2332-7812.

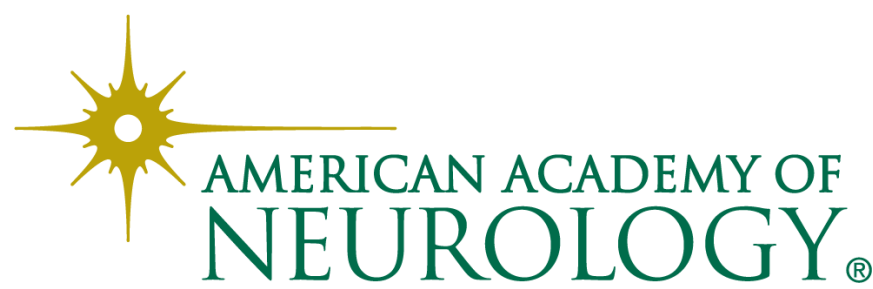




\section{Updated Information \& Services}

References

Citations

Subspecialty Collections

Permissions \& Licensing

Reprints including high resolution figures, can be found at: http://nn.neurology.org/content/7/6/e892.full.html

This article cites 12 articles, 1 of which you can access for free at: http://nn.neurology.org/content/7/6/e892.full.html\#\#ref-list-1

This article has been cited by 1 HighWire-hosted articles: http://nn.neurology.org/content/7/6/e892.full.html\#\#otherarticles

This article, along with others on similar topics, appears in the following collection(s):

\section{All Spinal Cord}

http://nn.neurology.org//cgi/collection/all_spinal_cord Cerebrospinal Fluid

http://nn.neurology.org//cgi/collection/cerebrospinal_fluid

Devic's syndrome

http://nn.neurology.org//cgi/collection/devics_syndrome

Multiple sclerosis

http://nn.neurology.org//cgi/collection/multiple_sclerosis

Information about reproducing this article in parts (figures,tables) or in its entirety can be found online at:

http://nn.neurology.org/misc/about.xhtml\#permissions

Information about ordering reprints can be found online: http://nn.neurology.org/misc/addir.xhtml\#reprintsus

Neurol Neuroimmunol Neuroinflamm is an official journal of the American Academy of Neurology.

Published since April 2014, it is an open-access, online-only, continuous publication journal. Copyright

Copyright $\odot 2020$ The Author(s). Published by Wolters Kluwer Health, Inc. on behalf of the American

Academy of Neurology.. All rights reserved. Online ISSN: 2332-7812.

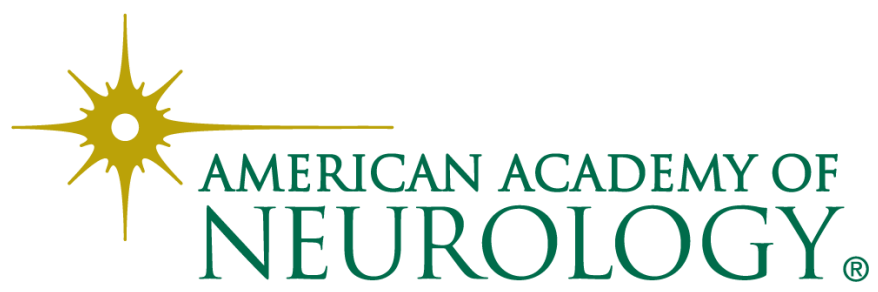

